\section{EXOPHTHALMIC GOITRE, AND OTHER CASES WITH ENLARGEMENT OF THE THYROID.}

BY EDWARD SQUIRE, M.D., M.R.C.P., PHYSICIAN TO ST. GEORGE'S AND ST. JAMES'S DISPENSARY, ASSISTANI PHYSICIAN TO THE NORTE LONDON HOSPITAL FOR DISEASES OF THE CHEST.

Case 1.-Mary Ann V--, aged fifteen years and two months, came under my care in January, 1884, when the following notes were taken. She is short for her age ( $5 \mathrm{ft} . \frac{1}{2}$ in.), and has never menstruated. The thyroid is enlarged, especially the right lobe, and this has been noticeable for three months. The neck is dark-brown in colour. The circumference of the neck over the thyroid is 12in. The eyes are slightly but obviously prominent, and her mother says she has noticed this for the past three years; she attributes' it to a fright. The heart's action is very violent; the apex beat is in the fifth space, but displaced to the left about an inch outside the nipple. There is a loud bruit de diable in the neck, and a hæmic murmur over the base of the heart. There are choreic movements in the left arm. A sense of constriction is felt in the throat on swallowing. In January of this year I had another opportunity of examining her. She has grown much taller, and menstruation began three montbs ago and is regular. There are still prominence of the eyes, enlargement of the thyroid, over-action of the heart, and a loud anæmic bruit in the neck; but she considers herself much better than when I first saw her.

CASE 2,-Emma W--, aged twenty-two, married two years (no children), was admitted in February, 1884, complaining of severe frontal headache and loss of appetite The thyroid is considerably enlarged, and has been so for three months; its size varies, being larger at the menstrual period, and then causes difficulty in swallowing. The neck now has a circumference of $15 \frac{1}{4} \mathrm{in}$. The eyes are sometimes painful, and project; this varies in extent with the variations in the size of the thyroid. There is excessive cardiac action and faintness sometimes occurs. Menstruation is irregular, scanty, and painful. There is a brownish pigmentation of the face.

CASE 3.-Matilda R- - aged twenty, single. This patient came under observation in July, 1882, with sore-throat. She is stout, but not strong. The thyroid is much enlarged, the circumference of the neck being quite 14 in. She has always had a "full neck," but for the last four or five years it has at times been noticeably swollen, especially since she came to London three years ago. The last time it was as large as it is now was eleven months ago. It has been very big for the last three or four days, and is worse in the morning when she wakes, when it seems high up in her neck, "up to her chin," and causes a sensation of choking. She is now unable to fasten the collar of her dress. Menstruation is regular, though scanty, and is now present. The heart's action is very weak, but she complains of pulsation in the thyroid. There is a loud venous bruit in the neck, and a soft apex murmur. On Aug. 16th she had a hysterical fit in the evening, and states that before she came to London such fits were of frequent occurrence.

CASE 4.--Mrs. B- aged twenty-two, married twelve months, and has one child three months old, which she has not been able to suckle. She was admitted in September, 1883 , for cough, and had deficient resonance over the apex of the left lung. The thyroid is enlarged, and has been so for three years. It varies in size, but no connexion is noticed between the increase of the neck and the menstrual period, which has been regular. There is a loud anæmic bruit in the neck, but no hæmic murmur in the chest.

CASE 5.-Eliza R -.., aged twenty, single. This patient, who is a stout well-grown girl, came under my care early this year complaining of lumbar pain. The thyroid is perceptibly enlarged, but causes no discomfort. The eyes are not prominent, and there is no over-action of the heart, though the patient complains of palpitation on exertion or after hurrying. There is a bruit de diable in the neck, and a soft hæmic apex murmur. Menstruation is regular, but scanty.

Remarks. - In the first two of the above cases we have examples of exophthalmic goitre, presenting singly no points of particular interest. They are described to be compared with those that follow. The first is a weli-marked case, though the age of the patient is below that at which the disease is most common. The second, though a slight case; has all the essential symptoms-exophthalmos, enlargement of the thyroid, and increased cardiac action. Both of these show indications of the dark colouration of the skin which has been noticed in Graves' disease, and which is supposed to bear an analogy with the pigmentation seen in Addison's disease, depending on alterations in the sympathetic nerves and ganglia. The remaining cases are wanting in some one or more of the cardinal symptoms of Graves' disease, and cannot be so named if the concurrence of the three mentioned above is essential. According to the usual definition they are so; but the late Dr. Hilton Fagge, speaking of exophthalmic goitre, ${ }^{1}$ warns us to be on the look-out for "slight cases in which one or two of the cardinal symptoms may be absent"; and Trousseau also insisted on this point, retaining the name "exophthalmic goitre" even when there is no exophthalmos. ${ }^{2}$ Case 3 has enlargement of the thyroid, but with no prominence of the eyes; and the cardiac action is weak, instead of being over strong. She is anæmic and hysterical-conditions often associated with exophthalmic goitre; here the thyroid increases in size at the catamenial period as in Case 2. Case 4 has thyroid enlargement, which varies in degree, but without the periodicity noticed in these two cases. She has, like the last cases of the series, palpitation, but no exophthalmos. From one point of view, Cases 3, 4 and 5 would appear to be examples of simple anæmia, in which enlargement of the thyroid occurs as one of the symptoms. The question then arises, whether the enlargement of the thyroid may not be a result of laxity of the tissues--a loss of tonicity-due to malnutrition from impoverished blood-supply. Over-action of the heart is also a frequent symptom in anæmia, and we have only the exophthalmos to explain to bring Graves' disease down from its position as a separate disease to the level of several other conditions which were loosely spoken of as diseases in themselves (such as albuminuria and jaundice), and are now recognised as merely symptoms. Begbie mentioned chlorosis and anæmia as powerful factors in inducing Graves' disease, but later writers consider it more probable that anæmia is a result. ${ }^{3}$ If so, it is sometimes a very early result, preceding some of the diagnostic symptoms of the disease. Graves himself thought that palpitation was the canse of the other two cardinal symptoms. Investigation with a view to discover a pathological cause for Graves disease, has produced no very definite result. Some observers have found degenerative conditions of the cervical sympathetic, which have been found wanting by others in necropsies on patients who were the subjects of undoubted exophthalmic goitre. It would appear, therefore, that the prominence of the eyes is not dependent on this condition of the sympathetics alone. Exophthalmos has been ascribed to repletion of the orbital vessels, as the bronchocele to a similar condition of the vessels of the thyroid, which repletion is due to loss of vascular tone. The loss of tone in the vessels is explained as a result of paralysis of the vaso-motornerves of the thyroid head, and neck. May not this depend on the want of healthy blood-supply to those nerve-centres in common with the rest of the tissues. In anæmia we have many signs that the tissues suffer from the supply of impoverished blood, Muscular weakness is common - weakness of nerve-control is shown by the easily induced palpitation and cold sweats from slight shocks or emotions in these patients, and by hysterical manifestations; and vascular want of tone explains the swelling of feet and ankles. Caleb Parry describes ${ }^{4}$ twelve cases of enlarged thyroid in women and one in a man, of which four of the female cases and that in the male he describes as associated with head symptoms. The remaining eight-of which the first, observed in 1786 , has her eyes "protruded from their sockets"-he speaks of as "enlargement of the thyroid gland in connexion with enlargement and palpitation of the heart"; and places them under the heading "Diseases of the Heart." Three occur in youngish unmarried women, and are associated with palpitation; the remainder, including that with exophthalmos, are in older women, and appear to be associated with heart disease. In one of the former the

\section{Principles of Medicine.}

2 Trousseau's Clinical Medicine. New Sydenham Society, 1868, vol, i. pp. 557. 3 Vide Ross: Diseases of the Nervous System.

4 Unpublished writings (1825). 
enlargement of the thyroid is attributed to a fright, and the tumour enlarges at the menstrual period. In all, throbbing of the carotids is noted. On these cases he makes the following comments: "Enlargements of the thyroid gland are not uncommon in femules about the period when the body is fully formed and the menses are in a sort of wavering state. After both these points are completely established, they generally disappear if the health is otherwise good......It is indeed true that these swellings occur most often and in the greatest degree in young females who have led sedentary lives, and who are of what are called relaxed and nervous habits." It has appeared to me that slight enlargement of the thyroid is not uncommon in anæmic and emotional women. If this occur without exophthalmos and palpitation, we look on it merely as a symptom of the general condition; but if all three are present in the same patient, we give a different name to the illness, and seek a different pathological cause. Advance iu science usually tends towards simplification in classification. Have we not in this case arrived rather at greater complexity?

\section{A CASE OF}

COMPOUND FRACTURE OF THE PATELLA; PARTIAL NECROSIS OF ONE OF THE FRAGMENTS ; RECOVERY WITH MOVABLE JOINT.

WITH NOTES OF NINETEEN OTHER CASES OF COMPOUND FRACTURE OF THE PATELLA.

BY G. R. TURNER, F.R.C.S. ENG.,

DEMONSTRATOR OF ANATOMY AND JOINT LECTURER ON PRACTICAL SURGERY AT ST. GEORGE'S HOSPITAL; VISITING SURGEON, SEAMEN'S HOSPITAI, GREENWICH.

F. S-, aged thirty-two, married, sustained a compound fracture of the left patella by striking his knee against the kerbstone. The fracture was transverse, and over it was a nearly horizontal contused wound two inches and a half long, which freely communicated with the cavity of the joint, which was filled with blood-clot; separation of fragments, half an inch to an inch. The joint was syringed out with carbolic lotion ( 1 in 20), and full aseptic precautions used in the dressing and subsequent treatment of the case, there being no drainage other than that permitted by the wound. The case ran an aseptic course. There was no fever whatever. The wound would have healed readily but for a small sinus leading down to an exposed piece of the lower fragment. A sequestrum came away from this six weeks after the injury. Cicatrisation was soon afterwards complete. The patient has attended as an out-patient. He can now (March 16th) walk about and fl ex his knee very nearly to a right angle. The fragments are separated by about three-quarters of an inch when the limb is in the extended position; by about an inch and a quarter when the knee is flexed. The lower fragment is widened and irregular; at the outer part the skin is slightly adherent.

This case, I think, presents several points of interest: the transverse direction of the fracture; the little separation of the fragments; the aseptic course, although a portion of one of the fragments necrosed; the question of the immediate treatment of the injury, and when and to what extent passive motion should have subsequently been employed. Compound fractures of the patella are very often comminuted or - -shaped, but both in Mr. Poland's cases in the fiftythird volume of the Medical and Chirurgical Transactions, and in some others $I$ have collected, it is far from uncommon to find the direction of the fracture transverse. The fragments in compound cases appears often to be less widely separated than in simple ones; this may be due to the escape of fluid from the wound, or from the fact that muscular action has less to do with the causation.

The question of the immediate treatment in this case resolved itself into one either of suturing the fragments or simply leaving the wound alone, for I suppose that in these days of aseptic surgery few surgeons would be found to recommend either excision or amputation to a healthy man of thirty-two with a clean, although contused, wound over a fractured patella. 1 decided to adopt an expectant plan of treatment rather than suture, for the following reasons: the fragments were not at all widely separated; the injury was nearly twenty four hours old when I first saw the patient, and the case was doing well; there was sufficient drainage already provided by the wound; 1 could not tell to what extent the fragments were already injured, and, consequently, whether they would bear the strain of wiring. From the subsequent necrosis it would appear that it was well they were not subjected to further mechanical injury. Passive motion was necessarily delayed until the separation of the sequestrum and the final union of the wound. When cicatrisation first occurred there was considerable prominence of a pointed fragment, and it seemed as if very slight violence would cause this to perforate the skin. I have on several occasions flexed the limb as far as I dared, but the fragments being as yet united by fibrous tissue, I have been afraid of stretching or rupturing the uniting band.

It may be interesting to compare the results of $\mathrm{Mr}$. Poland's cases, published in 1870 , with some recent cases which have occurred since the more general adoption of antiseptic surgery. In Mr. Poland's eighty-five cases the treatment adopted was "strapping where permissible, ice, leeches, irrigation; and in the suppurating stage fomentations and free incisions, opium, tonics, and support." Mr. Poland divides his cases into those with incised wounds, of which six recovered and two died; those with lacerated and contused wounds, of which thirty recovered and nine died; those associated with gunshot wounds, of which eighteen recovered and threedied; those associated with lesions of other bones, of which ten recovered and six died: total, eightyfour recoveries and twenty deaths. The joint suppurated in sixty-three cases, and of those that recovered thirty-one had more or less complete ankylosis. The above statistics, as Mr. Poland observes, probably give too favourable a view of the result of such treatment, as successful cases more often find their way into newspapers than the unsuccessful. This remark will apply with equal force to the cases I am about to relate.

Mr. Rouse has had two cases at St. George's Hospital. 1. Henry $R$, aged forty-seven, fell on to his right knee from a height of from twelve to fourteen feet, striking it against the pavement. There was a small lacerated wound over the patella, which was obliquely fractured across the centre. There was half an inch separation of the fragments, the lower of which consisted of two pieces, the external wound corresponding to a depression between them. Antiseptic precautions were used, and a ham splint applied. The temperature rose on the third day to $101^{\circ}$ in the evening, otherwise it was normal throughout. The patient recovered with a perfectly movable joint. 2. Edward $\mathrm{B}-$, aged thirty-three, fell from a cart on to his left knee, sustaining a compound comminuted fracture of the patella, over which was a circular contused wound, "which evidently communicates with the joint, although no synovia escapes." The wound was syringed out with carbolic lotion and dressed antiseptically with carbolic gauze. The temperature rose to $100^{\circ}$ in the evening, otherwise it was normal throughout the case. The patient recovered, with a movable joint, without any bad symptom whatever.

Mr. Pick read before the Clinical Society on Oct. 18th, $1875,{ }^{2}$ a case of extensive wound of the knee-joint treated by Lister's antiseptic method, in which the patella was divided by a circular saw and the condyle of the femur wounded. The joint was washed out with carbolic lotion ( 1 in 40). Suppuration followed, and the patient, a young man aged eighteen, recovered with firm bony ankylosis of the joint.

Mr. Finlay ${ }^{3}$ records a case of bullet-wound of the kneejoint in which the patella was " grooved" by the projectile, which finally lodged in the femur. The wound was washed out with carbolised water, the bullet extracted, and carbolic oil employed as a dressing. A little fever followed, but the patient, a man aged thirty-one, recovered with a perfect joint.

Mr. Wheelhouse $:^{4}-$ Margaret $M-$, aged twenty-four, fell some twelve feet on to her right knee. Clean transverse wound; fragments half an inch apart; no comminution except one small splinter from upper one. Wound enlarged; joint cleared of blood and washed out with 1 in 20 carbolic lotion. Fragments drilled and wired. No drainage of any kind. Full aseptic precautions. Wound ran an aseptic

2 Brit. Med. Jour., Oct. 16th, $1875 .{ }^{3}$ Ibid., vol. i., 1883. 